Correspondence: Varvara Solodovnikova, Republic Scientific and Practical Center of Pulmonology and Tuberculosis, 157, Dolginovsky trakt, 220053 Minsk, Belarus.

Tel. +375.29.3437751.

E-mail: varvaras@tut.by

Key words: New anti-tuberculosis drugs; fluoroquinolone resistance; rifampicin resistance; second-line injectable drug resistance; adverse drug safety monitoring.

Contributions: All the authors contributed to the conception of the study and protocol development. VS, DK, SS, IC, data collection and data entry; YS, AK, VS, DK, data analysis and interpretation; VS, AK, DK, first draft of the paper. All the authors reviewed the paper critically and gave final approval of the paper.

Conflict of interest: The authors declare no conflict of interest.

Funding: This study was funded by the United States Agency for International Development. The funding body had no role in study design, data collection and analysis, decision to publish, or preparation of the manuscript.

Ethics: Permission to access the data was obtained from the National Tuberculosis Programme in Belarus. Ethics approval was obtained from the Ethics Committee of the Republic Scientific and Practical Centre of Pulmonology and Tuberculosis in Belarus. Exemption from an ethics review was also received from the World Health Organization Research Ethics Review Committee based in Geneva, Switzerland (ERC.0003310/11.03.2020). A waiver of informed consent was granted by ethics review bodies, as the study collected and analysed de-identified routine recording and reporting data.

Disclaimer: The authors alone are responsible for the views expressed in this publication and they do not necessarily represent the decisions or policies of the World Health Organization.

Acknowledgements: The authors thank the national TB counterparts of the Republic Scientific and Practical Centre of Pulmonology and Tuberculosis of Belarus for defining research questions and providing data for this study, and the secretariat of the European TB Research Initiative (ERI-TB) at the WHO Regional Office for Europe for organizing the Structured Operational Research Training (SORT-TB) for eastern European countries supported by the USAID-WHO regional partnership project to End TB in eastern Europe. SORT-TB curriculum was an adaptation of the UNICEF/ UNDP/World Bank/WHO Special Programme for Research and Training in Tropical Diseases (TDR) SORT IT course (https://www.who.int/tdr/ capacity/strengthening/sort/en/) to the eastern European context.

Received for publication: 19 July 2020.

Accepted for publication: 31 July 2020.

${ }^{\circ}$ World Health Organization 2021.

All rights reserved. The WHO Regional Office for Europe has granted the Publisher permission for the reproduction of this article.

Licensee PAGEPress, Italy

Monaldi Archives for Chest Disease 2021; $91: 1646$

doi: 10.4081/monaldi.2021.1646

Open access statement: In accordance with WHO's open-access publication policy for all work funded by WHO or authored/co-authored by WHO staff members, the WHO retains the copyright of this publication through a Creative Commons Attribution IGO licence (http://creativecommons.org/licenses/by/3.0/igo/legalcode) which permits unrestricted use, distribution and reproduction in any medium provided the original work is properly cited.

\section{Effectiveness and safety of delamanid- or bedaquiline-containing regimens among children and adolescents with multidrug resistant or extensively drug resistant tuberculosis: A nationwide study from Belarus, 2015-19}

\author{
Varvara Solodovnikova ${ }^{1}$, Ajay M.V. Kumar ${ }^{2,3,4}$, \\ Hennadz Hurevich ${ }^{1}$, Yuliia Sereda ${ }^{5}$, \\ Vera Auchynka ${ }^{1}$, Dzmitry Katovich ${ }^{6}$, \\ Dzmitry Klimuk ${ }^{1}$, Aliaksandr Skrahin ${ }^{7}$, \\ Svetlana Setkina $^{8}$, Iryna Charnysh ${ }^{8}$, \\ Askar Yedilbayev $^{5}$, Alena Skrahina ${ }^{1}$
}

${ }^{1}$ Republic Scientific and Practical Center of Pulmonology and Tuberculosis, Minsk, Belarus; ${ }^{2}$ International Union Against Tuberculosis and Lung Disease, Paris, France; ${ }^{3}$ International Union Against Tuberculosis and Lung Disease, South-East Asia Office, New Delhi, India; ${ }^{4}$ Yenepoya Medical College, Yenepoya (Deemed to be University), Mangaluru, India; ${ }^{5}$ World Health Organization, Regional Office for Europe, Copenhagen, Denmark; ${ }^{6}$ Pulmonary Centre Klinikum Mittelbaden GmbH, BadenBaden, Germany; ${ }^{7}$ Belarussian State Medical University, Minsk, Belarus; ${ }^{8}$ Center for Examinations and Tests in Health Service, Minsk, Belarus 
(94\%) were resolved before the end of treatment. All patients culture-positive at baseline $(n=34)$ became culture-negative within three months of treatment. Median time to culture conversion was 1.1 months (interquartile range: 0.9-1.6). Two patients were still receiving treatment at the time of analysis. The remaining 38 patients successfully completed treatment. Among those eligible and assessed at $6(n=32)$ and 12 months $(n=27)$ post-treatment, no recurrences were detected. In conclusion, treatment of children and adolescents with MDR-TB and XDR-TB using bedaquiline and/or delamanid containing regimens was effective and had favourable safety profile. Achieving such excellent outcomes under programmatic settings is encouraging for other national tuberculosis programmes, which are in the process of introducing or scaling-up the use of these new drugs in their countries.

\section{Introduction}

Tuberculosis (TB) remains an important public health problem globally. In 2018, an estimated 10 million people fell ill with TB and 1.45 million people died due to it, making TB the leading cause of death among infectious diseases [1]. Drug resistance among TB patients is a major concern worldwide, which is threatening to reverse the gains made in TB control.

According to the World Health Organization (WHO), about half a million people developed Multidrug-Resistant TB (defined as resistance to both rifampicin and isoniazid) or RifampicinResistant TB (RR/MDR-TB) in 2018 and of them, 6.2\% had extensively drug-resistant tuberculosis (XDR-TB, with additional resistance to fluoroquinolones and second-line injectables), globally [1]. The highest proportions of RR/MDR-TB and XDR-TB were recorded in countries of eastern Europe [1]. There are huge gaps in the cascade of care of these patients. Globally, the treatment coverage was 32\% for MDR/RR-TB patients and 38\% for XDR-TB patients in 2018, while treatment success was $56 \%$ and $39 \%$ respectively (2016 cohort) [1].

Drug resistant TB also affects children and adolescents, who have historically faced great neglect in the TB world. It is estimated that between 25,000 and 32,000 children develop RR/MDR-TB annually and 1,200 of them develop XDR-TB [2-4]. Unfortunately, more than $95 \%$ of children with MDR-TB or XDR-TB are not diagnosed and treated and about $21 \%$ are likely to die [4]. There are no estimates of RR/MDR-TB burden among adolescents [5].

Belarus is a country in eastern Europe with high burden of drug resistant TB. Despite the overall decline in estimated TB incidence (from 58 per 100,000 population in 2014 to 31 in 2018), the burden of MDR/RR-TB remains high in 2018 at 37\% among new patients and $69 \%$ among previously treated patients [1]. About $12 \%$ of MDR-TB patients are estimated to have XDR-TB [6]. According to national statistics, the incidence rate of TB in children and adolescents decreased from 3 per 100,000 in 2014 to 1.1 per 100,000 in 2018. Among children with TB, the proportion with RR/MDR-TB in 2018 was $38 \%$ and half of them had XDR-TB. There was high treatment coverage for MDR-TB patients (93\%) in 2018 and the treatment success for 2016 cohort of patients was $67 \%$, higher than global average (56\%) [1].

To improve the treatment success among M/XDR-TB patients in children, new drugs such as bedaquiline and delamanid are recommended by WHO and other groups [7-10]. A systematic PubMed search revealed only four studies on this issue which include two case reports and two case series - with the collective experience of just 48 patients [11-15]. The results from these stud- ies are promising both in terms of safety and early effectiveness (in relation to culture conversion). Barring one case report, none of the studies reported final treatment outcomes and no study has reported on the magnitude of recurrence post-treatment.

While several clinical trials are underway, most do not include children $[16,17]$. Hence, learning from the real-world experiences of countries is crucial for progress and has been identified as one of the operational research priorities by the European TB Research Initiative [18]. Belarus has been at the forefront of using bedaquiline among children since 2015 and delamanid since 2016, long before the recommendations from the WHO were issued. This provided an opportunity to assess the treatment effectiveness and safety and contribute to the global evidence on this issue. In this study, we aimed to assess effectiveness and safety of bedaquiline and/or delamanid containing treatment regimens in a nationwide cohort of children and adolescents ( $<18$ years) with M/XDR-TB in Belarus. The specific objectives were to i) describe the spectrum of adverse drug events, their severity, timing of occurrence and management and ii) assess interim and final treatment outcomes and post-treatment recurrence.

\section{Materials and Methods}

\section{Study design}

This was a cohort study using routinely collected programme data.

\section{Study population and study period}

We included all eligible children and adolescents (aged $<18$ years) with RR/MDR-TB and XDR-TB started on treatment with bedaquiline and/or delamanid containing regimens in Belarus under the National Tuberculosis Programme (NTP) between September 2015 and September 2019. We included both bacteriologically confirmed and clinically diagnosed patients (i.e., children who were in contact with a confirmed index patient).

\section{Setting}

Belarus is an upper-middle income country in eastern Europe with a population of 9.5 million. Belarus consists of six regions and each region is subdivided into districts. The study was conducted in the Republican Scientific and Practical Centre of Pulmonology and Tuberculosis (RSPCPT), located in the capital city Minsk. This is the leading centre in the country for diagnosis and treatment of TB and other respiratory diseases. All children and adolescents with RR/MDR-TB in the country are treated at RSPCPT.

\section{Diagnosis of M/XDR-TB}

Children and adolescents with presumptive TB from the entire country are referred to the RSPCPT for diagnosis and treatment. Appropriate specimens (sputum or any other specimen including from an extrapulmonary TB) are subjected to sputum microscopy, Xpert MTB/RIF assay, Line Probe Assay (for both first- and second-line TB drugs), liquid and solid culture and phenotypic drug susceptibility tests (for both first- and second-line TB drugs). Some children without bacteriological confirmation were diagnosed based on a combination of chest radiography findings, clinical symptoms and history of close (household) contact with a case of confirmed MDR-TB or XDR-TB. 


\section{Treatment}

Based on the drug resistance pattern of the patient or index case, an individualized drug regimen was designed by the experts in the Republican MDR-TB Consilium. Composition of treatment regimens was based on inclusion of minimum of four effective drugs, with fluoroquinolones, bedaquiline and/or delamanid, linezolid, clofazimine and cycloserine as core drugs. Second-line injectables (capreomycin and amikacin), imipenem and amoxicillin/clavulanic acid and other drugs were added to treatment regimen when it was not possible to compose with minimum of four drugs from core list (Supplementary Table 1).

The treatment consisted of intensive phase for a period of 6-8 months and continuation phase for a period of 12-18 months. In case the resistance pattern allowed the use of short regimen (without injectables for a period of 9-12 months), the same was used after approval by the Republican MDR-TB Consilium [8]. Children and adolescents were hospitalized for a period of at least six months during the intensive phase. Once patients became culture negative and clinically stable, they were discharged from the hospital and referred to an outpatient health facility near to their place of residence for continuation of treatment and follow up. All patients were offered HIV testing and counselling. Those diagnosed as HIV-positive were started on antiretroviral therapy (ART) and cotrimoxazole preventive therapy (CPT), irrespective of the CD4 count or clinical staging.

\section{Follow-up and treatment monitoring}

The treatment response was assessed monthly, by performing sputum culture, smear microscopy and clinical examination (including weight gain). Chest radiography was performed every three months during the intensive phase and every six months during continuation phase. Blood tests were performed monthly to diagnose and timely treat adverse events including electrolyte abnormalities, anaemia, thrombocytopenia, leucopenia, liver and renal function tests. Electrocardiography (ECG) was performed every two weeks for first month and monthly until the end of therapy to identify prolongation of QTcF interval (adjusted using Fredericia formula) and arrhythmias. The common types of adverse events are shown in Supplementary Table 2.

\section{Grading of adverse events}

Grade refers to the severity of the adverse event. There are five grades as per Common Terminology Criteria for Adverse Events (CTCAE) [19]: i) Grade 1 (mild with no or minimal symptoms; requires only careful observation with no intervention; ii) Grade 2 (moderate requiring minimal, local or non-invasive intervention); iii) Grade 3 (medically significant event but not immediately lifethreatening; requiring hospitalization or prolongation of hospitalization; disabling; limiting the activities of daily living); iv) Grade 4 (life-threatening consequences requiring urgent intervention); v) Grade 5 (death related to $\mathrm{AE}$ ).

\section{Serious adverse event}

AEs are also classified as 'serious AE' or not as defined by The International Council for Harmonisation of Technical Requirements for Pharmaceuticals for Human Use (ICH). As per ICH guideline, a Serious Adverse Event (SAE) is any untoward medical occurrence that at any dose: i) results in death or ii) is life- threatening or iii) requires inpatient hospitalization or results in prolongation of existing hospitalization or iv) result in persistent or significant disability/incapacity or v) results in a congenital anomaly/ birth defect or vi) is a medically important event or reaction [20].
Based on the AE, if the offending drug was identified, one of the following actions was taken: close monitoring without changing the drug or dose, reducing the dose, temporary discontinuation of drug (or regimen) or permanent discontinuation of drug. Additional supportive treatment was provided, as appropriate.

\section{Recording}

All the patient details were recorded in the case files maintained at the hospital, the TB treatment cards and the TB register. All case definitions and outcomes were in line with WHO guidelines [21].

\section{Data variables and sources of data}

The variables related to the study objectives were extracted from the patient files maintained at the RSPCPT. These included the sociodemographic, behavioural and clinical data (diagnosis, treatment, adverse events and outcomes). Interim outcomes included culture conversion at six months (defined as two consecutive culture negative results obtained at least 30 days apart) and time to culture conversion. Final treatment outcomes were classified as successful (cured and treatment completed) and unsuccessful outcomes (death, loss to follow up, failure, not evaluated).

\section{Data entry and analysis}

The data were entered from the data sources mentioned above into a structured EpiData database created for the purpose. Double entry and validation were done to ensure quality of data. Analysis was done using $\mathrm{R}$ software version 3.5.2 ( ${ }^{\circ} \mathrm{R}$ Foundation for Statistical Computing, 2016). The sociodemographic and clinical profile of study participants and treatment outcomes was described using frequencies and proportions (for categorical variables) and mean and Standard Deviation (SD) or Median and Interquartile Range (IQR) for continuous variables, as appropriate. For analysis of adverse events, we took every event as the unit of analysis. The adverse events were summarized by type of event, severity and seriousness, timing of onset and duration, management and their outcome. We calculated the incidence rate of serious adverse events by dividing the number of events by the number of personmonths of observation.

\section{Results}

\section{Patient characteristics}

A total of 40 children and adolescents were included in the study. The mean age of patients was 15.6 (SD 1.7) years and 55\% were females (Table 1). While no one reported alcohol use, $15 \%$ were tobacco smokers. About one-third (30\%) of children and adolescents did not have a history of close contact with a tuberculosis patient. The majority of the patients (55\%) had comorbidities. Only two patients were HIV-positive, and both received ART and CPT. Most of the patients (93\%) had pulmonary TB and had bacteriologically confirmed TB (90\%). Half of the patients $(20,50 \%)$ had XDR-TB and resistance to fluoroquinolones or second line injectables was observed in 15 patients (38\%). A total of 20 patients $(50 \%)$ received delamanid and 21 (53\%) received bedaquiline, and one received both bedaquiline and delamanid concomitantly. All patients received bedaquiline and/or delamanid for 24 weeks, except one patient with XDR-TB who received delamanid throughout the entire duration of treatment (18 months) and two patients with XDR-TB who received bedaquiline for eight months. 
Table 1. Baseline demographic, behavioural and clinical characteristics of children and adolescents with multi/extensively drug-resistant tuberculosis who received bedaquiline or delamanid, 2015-2019, Belarus $(\mathrm{N}=40)$.

Demographic and behavioural characteristics $\quad$ n $\%$

\section{Demographic characteristics}

Age in years, mean (SD)

Age groups, years

Sex

Type of residence

\section{Behavioural characteristics}

Tobacco smoker

\begin{tabular}{ll} 
& No \\
Alcohol use & Yes \\
& No \\
\hline Clinical characteristics &
\end{tabular}

15.6

$10-14$

$15-17$

Male

Female

Urban

Rural

Yes 6

No

Yes

No

\begin{tabular}{cc}
8 & 20 \\
32 & 80 \\
18 & 45 \\
22 & 55 \\
20 & 50 \\
20 & 50 \\
\hline
\end{tabular}

$\begin{array}{cc}6 & 15 \\ 4 & 85 \\ 0 & 0 \\ 0 & 100\end{array}$

n

Body mass index, median $\left(25^{\text {th }}-75^{\text {th }}\right.$ percentiles)

Positive

$18.7(17.6-20.9)$

Comorbidities at baseline

Negative

$2 \quad 5$

Anaemia

38

Epilepsy

HIV

Myopia

Other comorbidities

History of TB treatment

New case

Previously treated

History of TB contact

Household member(s)

Other close contact(s)

None

Bacteriological confirmation

Yes

No

Baseline smear microscopy

Positive

Negative

Baseline culture
Baseline Xpert

Positive

Negative

Positive RR+

Negative

Type of drug resistance

RR/MDR

MDR (fluoroquinolones)

MDR (injectable)

XDR

TB localization

Pulmonary

Extrapulmonary

Both extrapulmonary and pulmonary

Presence of cavities

Yes

No

Drugs included in treatment regimen

Bedaquiline (Bdq)

Delamanid (Dlm)

Levofloxacin (Lfx)

Linezolid (Lzd)

Clofazimine (Cfz)

ycloserine ( $\mathrm{s}$ )

Terizidone (Trd)

Amikacyn (Am)

Capreomycin (Cm)

Prothionamide (Pto)

Imipenem/cilastatin (Imp)+ Amoxicillin/lavulanic acid (Amx/Clv)

Data are presented as n (\%) unless otherwise said; ART; antiretroviral therapy; HIV, human immunodeficiency virus; MDR, multi-drug-resistant; RR, rifampicinum resistance; TB, tuberculosis; XDR, extensively drugresistant; SD, standard deviation. 


\section{Adverse drug events}

Adverse events (AE) were reported in all the patients (Table 2 ). The number of AEs per patient ranged from 3 to 12 with a median of 5 (IQR: 3-8). A total of 224 AEs were recorded, most of which $(76 \%)$ were mild in nature. Only 10 AEs (4.5\%) were graded as severe and one AE was considered life-threatening. A total of $7(3 \%)$ AEs were classified as 'serious'. Modification in TB treatment regimen was done in 20 instances $(9 \%)-$ in 14 instances requiring a reduction in dose, five instances requiring temporary withholding and only one instance requiring permanent discontinuation of the suspected drug (linezolid was withdrawn after development of polyneuropathy). The details of management of all AEs are presented in Supplementary Table 3. The most common AEs were blood and lymphatic system disorders such as anaemia, eosinophilia and thrombocytopenia $(n=62$, $28 \%$ ) followed by cardiovascular disorders such as corrected QT

Table 2. Spectrum of adverse drug events and their severity in children and adolescents with multi/extensively drug-resistant tuberculosis who received bedaquiline or delamanid, 2015-2019, Belarus $(\mathrm{N}=40)$.

\begin{tabular}{|c|c|c|c|c|c|c|c|}
\hline & $\begin{array}{l}\text { Proportion of } \\
\text { patients with } \mathrm{AE} \text {, } \\
\text { n }(\%)^{\circ}\end{array}$ & $\begin{array}{c}\text { IR, per } 100 \\
\text { p-m }[95 \% \mathrm{CI}]\end{array}$ & $\begin{array}{l}\text { Total number of } \\
\text { AE, n }(\%)^{*}\end{array}$ & Mild & $\begin{array}{l}\text { AE gra } \\
\text { Moderate }\end{array}$ & $\begin{array}{l}\text { e, } n \\
\text { Severe }\end{array}$ & $\begin{array}{l}\text { Life- } \\
\text { threatening }\end{array}$ \\
\hline Eosinophilia & $22(55)$ & $2.9[1.9-4.4]$ & $23 \quad(10)$ & 12 & 9 & 2 & - \\
\hline Anaemia & $22(55)$ & $2.8[1.8-4.3]$ & $22 \quad(10)$ & 16 & 6 & - & - \\
\hline AST increased & $15(37.5)$ & $2.3[1.4-3.6]$ & $18 \quad(8)$ & 16 & 2 & - & - \\
\hline Cardiac disorders & $17(42.5)$ & $2.2[1.3-3.5]$ & $17 \quad(8)$ & 17 & - & - & - \\
\hline Corrected QT interval prolongation & $16(40)$ & $2.2[1.3-3.5]$ & $17 \quad(8)$ & 11 & 1 & 5 & - \\
\hline Creatinine increased & $15(37.5)$ & $1.9[1.1-3.2]$ & $15 \quad(7)$ & 13 & 2 & - & - \\
\hline ALT increased & $8(20)$ & $1.3[0.6-2.4]$ & $10 \quad(4)$ & 8 & 1 & 1 & - \\
\hline Hypocalcaemia & $10(25)$ & $1.3[0.6-2.4]$ & $10 \quad(4)$ & 7 & 2 & - & 1 \\
\hline Thrombocytopenia & $10(25)$ & $1.3[0.6-2.4]$ & $10 \quad(4)$ & 7 & 3 & - & - \\
\hline AP increased & $7(17.5)$ & $0.9[0.4-1.8]$ & $7 \quad(3)$ & 6 & 1 & - & - \\
\hline Bilirubin increased & $6(15)$ & $0.8[0.3-1.7]$ & $6 \quad(3)$ & 5 & 1 & - & - \\
\hline Hyperuricemia & $5(12.5)$ & $0.6[0.2-1.5]$ & $5 \quad(2)$ & 4 & 1 & - & - \\
\hline Hypomagnesaemia & $5(12.5)$ & $0.6[0.2-1.5]$ & $5 \quad(2)$ & 3 & 1 & 1 & - \\
\hline LDG increased & $5(12.5)$ & $0.6[0.2-1.5]$ & $5 \quad(2)$ & 5 & - & - & - \\
\hline Leukopenia & $5(12.5)$ & $0.6[0.2-1.5]$ & $5 \quad(2)$ & 5 & - & - & - \\
\hline Joint pain & $4(10)$ & $0.5[0.1-1.3]$ & $4 \quad(2)$ & 3 & 1 & - & - \\
\hline Headache & $3(7.5)$ & $0.4[0.1-1.1]$ & $3 \quad(1)$ & 3 & - & - & - \\
\hline Urea increased & $3(7.5)$ & $0.4[0.1-1.1]$ & $3 \quad(1)$ & 2 & 1 & - & - \\
\hline Other & $23(57.5)$ & $5.0[3.5-6.8]$ & $39 \quad(17)$ & 28 & 10 & 1 & - \\
\hline Total & $40(100)$ & $28.6[25.0-32.6]$ & $224(100)$ & 171 & 42 & 10 & $1^{\mathrm{c}}$ \\
\hline
\end{tabular}

'Denominator is the total number of patients; *denominator is the total number of adverse events. AE, adverse event; ALT, alanine aminotransferase; AP, alkaline phosphatase; AST, aspartate aminotransferase; IR, incidence rate; LDG, lactate dehydrogenase; p-m, person-months.

Table 3. Interim and final treatment outcomes in children and adolescents with multi/extensively drug-resistant tuberculosis who received bedaquiline or delamanid, 2015-2019, Belarus $(\mathrm{N}=40)$.

\begin{tabular}{|c|c|c|}
\hline Characteristics & $\mathrm{n} / \mathrm{N}$ & $\%$ \\
\hline $\begin{array}{l}\text { Interim treatment outcome } \\
\text { Culture negative at } 6 \text { months of anti-TB treatment }{ }^{\circ}\end{array}$ & $34 / 34$ & 100 \\
\hline Final treatment outcome & & \\
\hline $\begin{array}{l}\text { Treatment outcome evaluated } \\
\text { Treatment success } \\
\text { Cured } \\
\text { Treatment completed } \\
\text { Treatment failed } \\
\text { Died } \\
\text { Lost to follow-up }\end{array}$ & $\begin{array}{l}38 / 40 \\
38 / 38 \\
30 / 38 \\
8 / 38 \\
0 / 38 \\
0 / 38 \\
0 / 38\end{array}$ & $\begin{array}{c}95 \\
100 \\
79 \\
21 \\
0 \\
0 \\
0\end{array}$ \\
\hline $\begin{array}{l}\text { Recurrence } \\
\text { At } 6 \text { months* } \\
\text { At } 12 \text { months }\end{array}$ & $\begin{array}{l}0 / 32 \\
0 / 27\end{array}$ & $\begin{array}{l}0 \\
0\end{array}$ \\
\hline
\end{tabular}

'This analysis is restricted to patients who were culture-positive at baseline; *among patients who were eligible for 6-month follow-up assessment after completion of anti-TB treatment; ${ }^{\sharp}$ among patients who were eligible for 12-month follow-up assessment after completion of anti-TB treatment; TB, tuberculosis. 
interval prolongation and other cardiac abnormalities $(n=43$, $19 \%)$ and hepatobiliary disorders $(n=34,15 \%)$ (Figure 1, Supplementary Table 4). The incidence rate of AEs was highest during the first two months of treatment (Figure 2,
Supplementary Table 5) and declined as the treatment progressed. Most of the AEs (94\%) were resolved before the end of the treatment. The median (IQR) time to resolution of AE was 84 (IQR: 31-177) days.

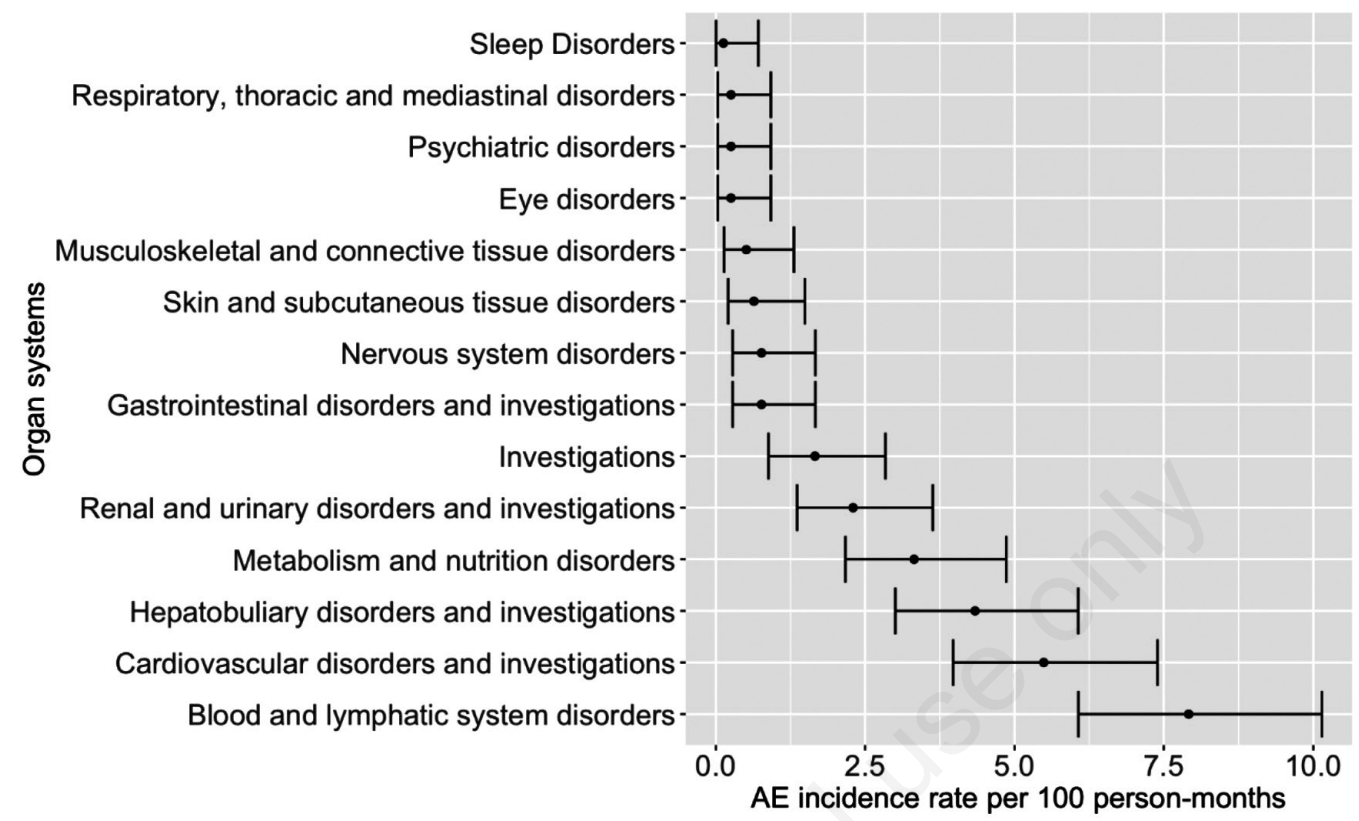

Figure 1. Incidence rate of adverse drug events by organ systems in children and adolescents with multi/extensively drug-resistant tuberculosis who received bedaquiline or delamanid, 2015-2019, Belarus $(\mathrm{N}=40)$.

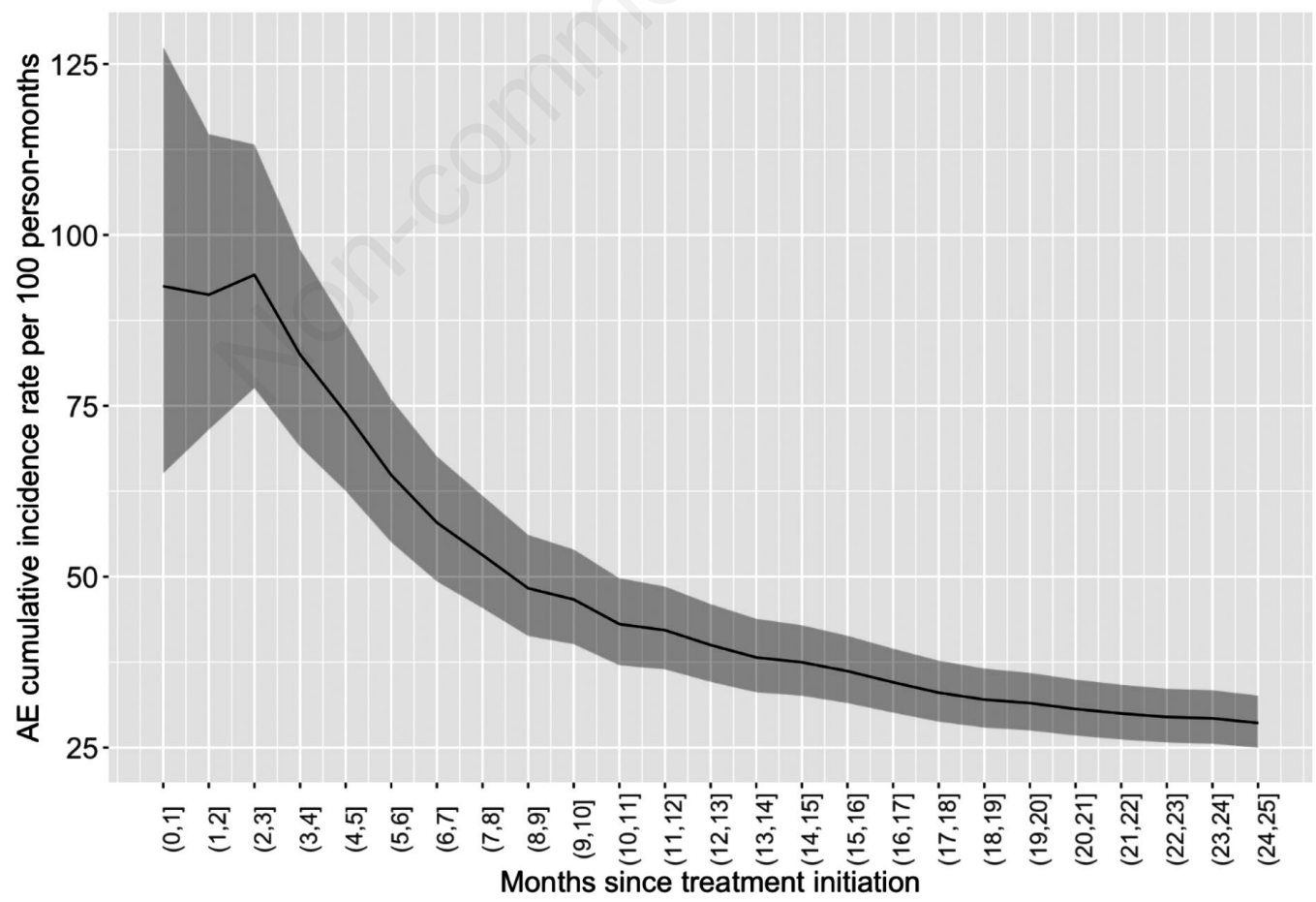

Figure 2. Cumulative incidence rate of adverse drug events along the follow up in children and adolescents with multi/extensively drugresistant tuberculosis who received bedaquiline or delamanid, 2015-2019, Belarus $(\mathrm{N}=40)$. Square brackets mean the end point is included, and round parentheses mean it is excluded; for example $(1,2]$ means above the one and below or equal two. Grey ribbon indicates $95 \%$ confidence interval; $\mathrm{AE}$, adverse events. 


\section{Treatment outcomes}

All patients who were culture-positive at baseline $(n=34)$ achieved culture conversion during first six months of treatment (Table 3). The median time to culture conversion was 1.1 months (IQR: 0.9-1.6; Range: 0.4-2.8). Of 40 patients, two were still on treatment by the time of analysis, thus, were excluded from analysis of final outcomes. The remaining 38 patients completed treatment successfully. No deaths, failures or losses to follow-up were found. Among those eligible and assessed at 6- and 12-months post-treatment, no recurrences were detected (Table 3 ).

\section{Discussion}

This is the first study from Belarus reporting on effectiveness (final treatment outcomes and post-treatment recurrence) and safety of bedaquiline and delamanid containing regimens in children and adolescents with RR/MDR-TB and XDR-TB. This is the largest cohort of children and adolescents ever reported worldwide, thus adding to the limited global evidence on this issue.

There were two key findings. First, we found that treatment regimens were highly effective - with a $100 \%$ culture conversion within the first three months of treatment, $100 \%$ treatment success at the end of treatment and no recurrence after 6- and 12-months post-treatment. These results are hugely promising to consider the fact that this cohort was treated under programmatic conditions and nearly $90 \%$ of the patients had XDR-TB or pre-XDR-TB at baseline. Second, we found that the regimens were safe and welltolerated. Although all patients experienced AEs, most of AEs were mild and did not require any intervention. Only one episode of serious $\mathrm{AE}$ was reported that led to permanent discontinuation of the suspected agent. Safety profile of new drugs in the paediatric cohort was similar to the adult population [22]. These findings are reassuring and are in line with the results of previous case reports and case series on this issue [11-15].

Our study had several strengths. First, this was a nationwide cohort and included all children and adolescents treated with new drugs in Belarus between 2015 and 2019. Thus, the findings are likely to be generalizable to Belarus and other countries in the eastern Europe having similar patient profiles. Second, we had an active and meticulous system of identification, recording and reporting of AEs - both during hospital admission and ambulatory care. Thus, it provides complete information of spectrum of AEs to be expected by NTPs when treating patients with the new drugs and be prepared accordingly. One limitation was that our sample size was small which precluded the conduct of multivariable analysis to assess the factors associated with AEs.

The findings have major implications for NTPs of countries in the WHO European Region and elsewhere grappling with an epidemic of drug resistant tuberculosis. Globally, the treatment outcomes among RR/MDR-TB and XDR-TB patients have been poor at $56 \%$ and $39 \%$ respectively [1]. Similar results are reported from the WHO European region too (57\% and 39\% respectively) and these are way below the target of $75 \%$ to be achieved $[23,24]$. This experience from Belarus of using new drugs under programme settings provides new hope that the target of $75 \%$ can be achieved (and improved upon), if countries commit to introduce and scaleup the use of new drugs. Rapid culture conversion associated with the new drugs may also help in cutting the chain of transmission of drug resistant tuberculosis in the community.

Our results lend confidence to the NTPs' transition to modi- fied, all-oral shorter MDR-TB treatment regimens in children. There are multiple challenges involved with use of new drugs which include limited availability and access, budgets to cover the high costs, absence of regulatory in-country approvals for use of these drugs, lack of appropriate tools (clinical guidelines and training plans) and mechanisms for active drug safety monitoring [13]. If these challenges are addressed, we may move a step closer to achieving the dream of ending TB in the WHO European region.

\section{Conclusions}

In conclusion, we found that treatment of children and adolescents with RR/MDR-TB and XDR-TB with bedaquiline and delamanid containing regimens was effective and had a favourable safety profile. Achieving such excellent outcomes under programmatic settings is encouraging for other NTPs who are in the process of introducing or scaling-up the use of these new drugs in their countries.

\section{References}

1. WHO. Global tuberculosis report 2019. Geneva: World Health Organization; 2019:1-297. Available from: https://www.who. int/tb/publications/global_report/en/

2. Dodd PJ, Sismanidis C, Seddon JA. Global burden of drugresistant tuberculosis in children: a mathematical modelling study. Lancet Infect Dis 2016;16:1193-201.

3. Jenkins HE, Tolman AW, Yuen CM, et al. Incidence of multidrug-resistant tuberculosis disease in children: systematic review and global estimates. Lancet 2014;383:1572-9.

4. Jenkins HE, Yuen CM. The burden of multidrug-resistant tuberculosis in children. Int J Tuberc Lung Dis 2018;22:3-6.

5. García-Basteiro AL, Schaaf HS, Diel R, et al. Adolescents and young adults: a neglected population group for tuberculosis surveillance. Eur Respir J 2018;51.

6. Skrahina A, Hurevich H, Zalutskaya A, et al. Multidrug-resistant tuberculosis in belarus: the size of the problem and associated risk factors. Bull World Health Organ 2013;91:36-45.

7. Harausz EP, Garcia-Prats AJ, Seddon JA, et al. New and repurposed drugs for pediatric multidrug-resistant tuberculosis. practice-based recommendations. Am J Respir Crit Care Med 2017;195:1300-10.

8. WHO. Consolidated guidelines on drug-resistant tuberculosis treatment. Geneva: World Health Organization; 2019:1-104. Accessed on: 2019 Oct 4. Available from: https://www.ncbi. nlm.nih.gov/books/NBK539517/

9. The Sentinel Project for Pediatric Drug-Resistant Tuberculosis. Management of multidrug-resistant tuberculosis in children: a field guide. Fourth edition. 2018:1-77. Accessed on: 2020 Jul 3. Available from: http://sentinel-project.org/wpcontent/uploads/2019/02/Updated_DRTB-Field-Guide-2019V3.pdf

10. Migliori GB, Tiberi S, Zumla A, et al. MDR/XDR-TB management of patients and contacts: challenges facing the new decade. The 2020 clinical update by the global tuberculosis network. Int J Infect Dis 2020;92:S15-25.

11. Esposito S, D'Ambrosio L, Tadolini M, et al. ERS/who tuberculosis consilium assistance with extensively drug-resistant 
tuberculosis management in a child: case study of compassionate delamanid use. Eur Res J 2014;44:811-5.

12. Esposito S, Bosis S, Tadolini M, et al. Efficacy, safety, and tolerability of a 24-month treatment regimen including delamanid in a child with extensively drug-resistant tuberculosis: a case report and review of the literature. Medicine (Baltimore) 2016;95:e5347.

13. Tadolini M, Garcia-Prats AJ, D'Ambrosio L, et al. Compassionate use of new drugs in children and adolescents with multidrug-resistant and extensively drug-resistant tuberculosis: early experiences and challenges. Eur Respir J 2016;48:938-43.

14. Achar J, Hewison C, Cavalheiro AP, et al. Off-label use of bedaquiline in children and adolescents with multidrug-resistant tuberculosis. Emerg Infect Dis 2017;23:1711-3.

15. D'Ambrosio L, Centis R, Tiberi S, et al. Delamanid and bedaquiline to treat multidrug-resistant and extensively drugresistant tuberculosis in children: a systematic review. J Thorac Dis 2017;9:2093-101.

16. Clinicaltrials.gov. Evaluating newly approved drugs for multidrug-resistant tb (endtb): clinicaltrials.gov identifier: nct02754765. Accessed on: 2020 Jul 2. Available from: https://clinicaltrials.gov/ct2/show/NCT02754765.

17. Clinicaltrials.gov. Pragmatic clinical trial for a more effective concise and less toxic mdr-tb treatment regimen(s) (tb-practecal): clinicaltrials.gov identifier: nct02589782. Accessed on: $2020 \mathrm{Jul}$ 2. Available from: https://clinicaltrials.gov/ct2/show/ NCT02589782.

18. WHO Regional Office for Europe. Defining tuberculosis the research agenda for the who european region: a study report of the european tb research initiative. Copenhagen, Denmark; 2019:1-23. Accessed on: 2019 Nov 21. Available from: https://apps.who.int/iris/bitstream/handle/10665/327085/9789 289054317-eng.pdf? sequence $=1$ \&isAllowed $=y$

19. US Department of Health and Human Services, National Institutes of Health, National Cancer Institute. Common Terminology Criteria for Adverse Events (CTCAE) version 5.0. 2017. Accessed on: 2019 Nov 23. Available from: https://ctep.cancer.gov/protocoldevelopment/electronic_applications/docs/CTCAE_v5_Quick_Reference_8.5x11.pdf.

20. European Medicines Agency. ICH e2d post-approval safety data management. 2003:1-8. Accessed on: 2020 Jul 31. Available from: https://www.ema.europa.eu/en/ich-e2d-postapproval-safety-data-management.

21. WHO. Definitions and reporting framework for tuberculosis 2013 revision. Geneva: World Health Organization; 2013.

22. Borisov S, Danila E, Maryandyshev A, et al. Surveillance of adverse events in the treatment of drug-resistant tuberculosis: first global report. Eur Respir J 2019;54:1901522.

23. WHO Regional Office for Europe, European Centre for Disease Prevention and Control. Tuberculosis surveillance and monitoring in Europe 2020. Copenhagen, Denmark: WHO; 2019:1-164. Accessed on: 2020 Jul 2. Available from: https://www.euro.who.int/en/publications/abstracts/tuberculosis-surveillance-and-monitoring-report-in-europe-2020

24. WHO Regional Office for Europe. Tuberculosis action plan for the who european region 2016-2020. Copenhagen, Denmark: WHO; 2015. Accessed on: 2019 Nov 21. Available from: http://www.euro.who.int/_data/assets/pdf_file/0007/283804/ 65wd17e_Rev1_TBActionPlan_150588_withCover.pdf?ua $=1$ 